\title{
Derivation of a Closed Form Expression for Estimating the Reduced Flow Rate for Pressure Driven Rarefied Gas Flow Through Circular Nano/Micro Pores
}

\author{
S. A. Hashemifard ${ }^{a^{*}} \&$ T. Matsuura ${ }^{b}$ \\ asustainable Membrane Technology Research Group (SMTRG), Faculty of Petroleum, \\ Gas and Petrochemical Engineering (FPGPE), Persian Gulf University (PGU), P.O. \\ Box 75169-13798, Bushehr, Iran \\ ${ }^{b}$ Department of Chemical and Biological Engineering, University of Ottawa, 161 \\ Louis Pasteur St., Ottawa, ON, Canada K1N 6N5
}

Submitted: 11/4/2021. Revised edition: 7/5/2021. Accepted: 7/5/2021. Available online: 15/7/2021

\begin{abstract}
In this paper, a new model to predict the gas flow rate through short tubes under rarefied condition based on the sigmoidal bahaviour of gas reduced flow rate $(W)$ versus the rarefaction parameter $(\delta)$ under rarefied condition was developed. The data produced by Varoutis et al. via Direct Simulation Monte Carlo (DSMC) method were utilised to obtain the model coefficients as functions of tube length to radius $(\omega)$ and pressure ratio $(P r)$. Then, the model was tested against the published experimental data.There was a high degree of agreement between the model predictions and the experimental data. Moreover, the new model was capable to predict the reduced flow rate of rarefied systems, not only at free molecular region and hydrodynamic region, but also at transition region, hence covering all the Knudsen number domain within the utilised data. Therefore, the proposed model was capable to make predictions as well as meet all the criteria of the rarefied gas flow within the following conditions: $0<\operatorname{Pr}<0.9,0.01<\delta<1000$ and $0.0<\omega<20$. Thus, the proposed model provides a useful tool to make a valid prediction of the rarefied gas flow behavior in a wide range of gas transport regime.
\end{abstract}

Keywords: Modelling, rarefaction, short tubes, S-shaped

\subsection{INTRODUCTION}

Selecting a system possessing nano/micro pores under rarefaction condition for a particular application one needs an in depth knowledge about the gas penetration mechanism, and an accurate undestanding of the system pore size and the length to diameter ratio. A number of systems possess tubes or pores having small sizes and finite lengths under low pressure, wherein the system charactersitic length is compatible with the gas mean free path. Whithin such systems, we are dealing with a combination of both continuum regime and free molecular regime, [1]. Thus, a non-continuous mechanics is applied. Some of the well-known applications of rarefied systems include: porous membrane and porous media, instrumentation, microelectromechanical systems, microchannel networks, microelectronics etc. [2].

A number of methods can be considered for predicting the behaviour of such systems as well as estimating their structural parameters. Simply put, 
three techniques can be accounted: i) Analytical method, ii) Emperical equation method and iii) Direct Simulation Monte Carlo (DSMC) method. Sreekanth reported the observations of rarefied gas transition flow as an extention of Liepmann's work through a short tube in which the length was shifted to a an orifice at vacuum condition i.e. free molecular regime. The Knudsen number ranged whithin a limited interval [3, 4]. Fujimoto and Usami investigated the Rarefied gas flow through short tubes experimentally and the flow rate was measured for a broad range of Knudsen number ranging from 0.0002 to 50, which finally an empirical equation was proposed. The equation yield the results as a function of Knudsen number, length to diameter and pressure ratios. Practically speaking, their model requires a cumbersome procedure to estimate the flow rate [5]. Moreover, Sreekanth [4], Fujimoto and Usami [5], Jitschin et al. [6], Barashkin [7] and Marino [8] made efforts to provide a number of valuable experimental data presenting rarefied gas flow. Because of scarcity of the experimental data covering a full range of pressure and length to diameter ratios as well as Knudsen number, the vital role of the DSMC data is revealed. Considerable prominant research have been carried out applying DSMC to describe the short tube systems under rarfied conditions, best examplifoed by Alexeeno et al. [9, 10], Lilly et al. [11], Varoutis et al. [12] and Varoutis et al. [13].

However, the results of the linearized Boltzmann equation [14] has given good results, the application of such techniques is practically limited. The valuable predictions arisen from the numerical DSMC suffer from a cumbersome computation with a considerable computational cost ranging from a few hours to a few weeks. Accordingly, for easy and practical computational purposes, it seems vital to deal with a direct model based on a simple empirical equation. In fact, it has been repeatedly reported in many engineering and environmental works that the system's output can follow the S-shaped (or sigmoid) function of a variable, either in the arithmetic or semi-logarithmic coordinates [15]. Some common examples are as follows: population growth, competition among different species in biology, ecology and technology domains, technological forecasting, breakthrough curves of penetration of one species in a media, etc. [16].

Accordingly, for easy and practical computational purposes, e.g. pore size prediction in porous media such as oil/gas reservoir cores or porous membranes, it seems crucial to deal with a direct and explicit model based on a simple empirical equation. A glance through the experimental data of the reduced flow rate versus Knudsen number or rarefaction parameter under rarefied condition reveals that the trend follows quite an S-shaped function. Therefore, in this work, a closed form expression empirical model is proposed based on the s-shaped behavior of the rarefied gas to approximate the reduced gas flow rates for pressure driven flow through short circular tubes in a wide range of the involved parameters. The data produced by Varoutis et al. via DSMC $[12,13]$ were used to obtain the coefficients involved in the equation as a function of $\omega$ and Pr. Then the proposed model was tested against the experimental data $[4,5,6,7,8]$ from the open literature. The proposed model was capable to make prediction in the following ranges of the parameters: $0<\operatorname{Pr}<0.9,0.01<\delta<1000$ and $0.0<\omega<20$. 


\subsection{THEORETICAL BACKGROUND}

The reduced flow rate $(W)$ is defined by Equation (1) through a short tube [17]:

$$
W=\frac{\dot{\mathrm{m}}}{\dot{\mathrm{m}}_{0}}
$$

that $\dot{m}(\mathrm{~kg} / \mathrm{s})$ is mass flow rate and as a reference, the flow rate through an orifice at the free molecular regime (into vacuum), is given by [12]:

$$
\dot{m}_{0}=\frac{\sqrt{\pi} r_{p}^{2} P_{0}}{v_{m}}
$$

that, $r p(\mathrm{~m})$ is the tube size, and the pressure ratio is:

$$
P_{r}=\frac{P_{1}}{P_{0}}
$$

where the upstream and downstream pressures are shown by $P_{0}$ and $P_{1}(\mathrm{~Pa})$, respectively. The most prabable gas velosity, $v_{m}(\mathrm{~m} / \mathrm{s})$ is defined as follows [18]:

$$
v_{m}=\left(\frac{2 R T_{0}}{M}\right)^{0.5}
$$

here, the upstream absolute temperature $(\mathrm{K})$ is shown by $T_{0}, R$ is the universal gas constant $(8.314 \mathrm{~J}$ mol-1 $\mathrm{K}-1)$ and $M$ is the molecular weight $(\mathrm{kg} / \mathrm{mol})$.
The rarefaction parameter $(\delta)$, is applied as an important criteria to discriminate the flow regimes [19]:

$\delta=\frac{\sqrt{\pi}}{2 \mathrm{Kn}}$

That $K_{n}$ is the Knudsen number defined as :

$$
K_{n}=\frac{\lambda}{r_{p}}
$$

$\lambda$ is the gas mean free path $(\mathrm{m})$ related to the $\mu_{0}$ the gas viscosity (Pa.s) by [18]:

$\lambda=\frac{\mu_{0} v_{m}}{P_{0}}$

a combination of Equations (5) to (7) gives the rarefaction parameter as:

$$
\delta=\frac{r_{p} P_{0}}{\mu_{0} v_{m}}
$$

subscript zero denotes the upstream condition. From Equation (5), as $K_{n}$ approaches infinity, $\delta$ approaches zero and we are in the free molecular region, while as $K_{n}$ approaches zero, $\delta$ approaches infinity, and we are in the hydrodynamic region. According to the Knudsen number or rarefaction parameter, various regimes can be described in micro/nano pores. The criteria proposed by Roy et al. [17, 20] using Knudsen number is employed in the present work for rarefaction parameter as well, as shown in Table 1. 
Table 1 Categorizing different flow regimes in micro/nano tubes based on the Knudsen number and the rarefaction parameter [20]

\begin{tabular}{|c|c|c|c|}
\hline Characteristics & Flow regime & $\begin{array}{l}\text { Knudsen number } \\
\text { limit }\end{array}$ & $\begin{array}{l}\text { Rarefaction } \\
\text { parameter limit }\end{array}$ \\
\hline Molecule-wall collision & Free molecular & $\mathrm{Kn} \geq 10$ & $\delta \leq 0.1$ \\
\hline $\begin{array}{l}\text { Transition from Molecule- } \\
\text { wall collision to molecule- } \\
\text { molecule collision }\end{array}$ & Transition & $0.1 \leq \mathrm{Kn}<10$ & $0.1<\delta \leq 10$ \\
\hline \multirow{2}{*}{ Molecule-molecule collision } & $\begin{array}{l}\text { Slip phenomena } \\
\text { (slip on the wall) }\end{array}$ & $0.001<\mathrm{Kn}<0.1$ & $10<\delta<1000$ \\
\hline & $\begin{array}{l}\text { Hydrodynamic } \\
\text { (friction on the wall) }\end{array}$ & $\mathrm{Kn} \leq 0.001$ & $\delta \geq 1000$ \\
\hline
\end{tabular}

It is well known that [13], the reduced flow rate varies as a function of $\delta$ and Pr. Moreover, the tube length to radius ratio $(\omega)$ is another quantity that is also used to describe the flow behavior.

$$
\omega=\frac{l_{p}}{r_{p}}
$$

that $l_{p}(\mathrm{~m})$ is the short tube length.

Before presenting a new model, it is worth reviewing the previously proposed models in brief to show their application ranges and limitations. When we are dealing with a high vacuum condition in the sink $\left(P_{r}=0\right)$, and experiencing the free molecular region (i.e. $\delta \rightarrow 0$ ) the following formula was used by Steckelmacher to predict the reduced flow rate for short tubes [21]:

$$
W=\frac{1}{1+\omega / 2}
$$

Which means $W$ approaches the transmission probability. It was shown by Sharipov that when the rarefaction parameter tends toward zero (free molecular region) and $\omega$ approaches zero (orifice), $W$, as a limit approaches 1-Pr [19]. Fujimoto and Usami carried out a vast experimental study on short tubes with different $\omega$ and in a broad range of Knudsen numbers $(2 \times 10-4$ to 50) for high vacuum condition $\left(P_{r}<0.01\right)$. Finally they proposed the following empirical model:

$$
\frac{F}{F_{f m 0}}=\frac{0.4733+H \sqrt{1 /\left(a R_{e}\right)^{c}}}{1+I /\left(a R_{e}\right)^{c}+K /\left(a R_{e}\right)^{2 c}} b+\frac{F_{f m}}{F_{f m 0}}
$$

$$
\begin{aligned}
& H=1.31\left(\frac{F_{f m}}{F_{f m 0}}\right)^{16} \\
& R_{e}=0.501\left(\frac{1}{K_{n}}\right)\left(\frac{F}{F_{f m 0}}\right)
\end{aligned}
$$

Where $R_{e}$ is the Reynolds number and $\mathrm{I}=8.537$ and $\mathrm{K}=3.599$. To calculate $F_{f m}$, they recommended to use the equation proposed by Henning [5]. The readers can refer to Table 2 of reference [5] for constants $a, b$ and $c$. As they have admitted, it is obvious that a cumbersome iterative procedure is required to estimate $W$. The extrapolation of Fujimoto and Usami model [22] for an orifice at high vacuum $\left(\omega=0\right.$ and $\left.P_{r}=0\right)$ yields: 


$$
W=1+\frac{0.4733+0.6005 / \delta}{1+4.559 / \delta+3094 / \delta^{2}}
$$

This equation gives good results for $0<\delta<20$.

Sreekanth [4] proposed a formula for more general cases as:

$$
W=\frac{1-P_{r}}{1+\omega / 2}\left[1.038+\frac{1+P_{r}}{5 \sqrt{\pi}} \delta\right]
$$

Equation (15), represents a linear relationship between $W$ and $\delta$, therefore the predictions are limited to the lower value of $\delta$ where the flow is mostly governed by the free molecular regime. Sharipov reported the following formula for $W$ in free molecular regime (i.e. $\delta<1$ ) for an orifice as well [19]:

$$
W=\left(1-P_{r}\right)(1+A \delta)
$$

Comparing the two last equations, applying some simplifications, one can reduce Equation (15) to Equation (16), where:

$$
\mathrm{A} \approx \frac{1+\mathrm{P}_{\mathrm{r}}}{5 \sqrt{\pi}}
$$

\subsection{RESULTS AND DISCUSSION}

\subsection{The Proposed Model}

As mentioned above this paper aims to derive a group of analytical equations that allow to reproduce the DSMC of Varoutis et al., since the DSMC enables to calculate $\mathrm{W}$ in the largest ranges of independent variables but requires a large amount of computation time.

From DSMCW is known to be a function of three independent variables, i.e. $\delta, \omega, \operatorname{Pr}$.

$\mathrm{W}=\mathrm{f}(\delta, \omega, \operatorname{Pr})$

and for a given set of $P_{r}$ and $\omega W$ is an $\mathrm{S}$-shaped function of $\delta$.

There are several equations to describe S-shaped functions, as shown in Table 3, and the best one will be chosen among these equations in the following way. It should be noted that each equation involves 3 parameters $\alpha, \beta$ and $\gamma$.

1) For arbitrarily chosen two sets of $P_{r}$ and $\omega\left(\left(P_{r}=0.1, \omega=0.1\right)\right.$ and $\left(P_{r}=\right.$ $0.1, \omega=0.5)), W$ is obtained from DSMC [12] as a function of $\delta$.

2) For each equation given in Table 3 , three parameters $\alpha, \beta$ and $\gamma$ are optimized, so that $W$ values calculated by the equation becomes closest to those generated by DSMC.

3) Then, $E_{r}$ (difference between the values obtained from the equation and those from DSMC) is calculated. $\quad \alpha, \beta, \gamma$, and $E_{r}$ so obtained are also shown in Table 3.

4) Finally, the equation that gives the smallest $E_{r}$ is chosen.

It is obvious from Table 2 that DSMC values are best fit by the following Equation (19).

$$
W=-\frac{\alpha}{\delta}\left(1-e^{-\beta \delta}\right)+\gamma
$$


Table 2 Preliminary evaluation of several S-shaped function

\begin{tabular}{|c|c|c|c|c|c|c|c|c|}
\hline \multirow[b]{2}{*}{ S-shaped function } & \multicolumn{4}{|c|}{$\omega=0.1$} & \multicolumn{4}{|c|}{$\omega=5.0$} \\
\hline & $\alpha$ & $\beta$ & $\gamma$ & $\begin{array}{c}\text { Abs.E } \\
\text { r. }\end{array}$ & $\alpha$ & $\beta$ & $\gamma$ & $\begin{array}{c}\text { Abs.E } \\
\text { r. }\end{array}$ \\
\hline $\begin{array}{c}\text { Gompertz function: } \\
\mathrm{y}=\alpha \mathrm{e}^{-\beta \mathrm{e}^{-\gamma \delta}}\end{array}$ & $\begin{array}{c}1.509 \\
79\end{array}$ & $\begin{array}{c}0.551 \\
56\end{array}$ & $\begin{array}{c}0.231 \\
94\end{array}$ & 1.00 & $\begin{array}{c}1.243 \\
59\end{array}$ & $\begin{array}{c}1.469 \\
4\end{array}$ & $\begin{array}{c}0.042 \\
17\end{array}$ & 4.77 \\
\hline $\begin{array}{l}\text { Normal growth function: } \\
\qquad \mathrm{y}=\frac{\alpha}{\beta+\mathrm{e}^{-\gamma \delta}}\end{array}$ & $\begin{array}{c}2.075 \\
13\end{array}$ & $\begin{array}{c}1.374 \\
26\end{array}$ & $\begin{array}{c}0.269 \\
52\end{array}$ & 1.19 & $\begin{array}{c}0.176 \\
84\end{array}$ & $\begin{array}{c}0.219 \\
5\end{array}$ & $\begin{array}{c}0.038 \\
6\end{array}$ & 6.12 \\
\hline S-shaped function from [15] & & & & & & & & \\
\hline $\mathrm{y}=-\frac{\alpha}{\delta}\left[1+(\mathrm{t}-1) \mathrm{e}^{-\beta \delta}\right]$ & $\begin{array}{c}0.517 \\
03\end{array}$ & $\begin{array}{l}0.265 \\
78\end{array}$ & $\begin{array}{c}1.504 \\
3\end{array}$ & 0.51 & $\begin{array}{c}0.945 \\
67\end{array}$ & $\begin{array}{c}0.025 \\
3\end{array}$ & $\begin{array}{c}1.249 \\
67\end{array}$ & 2.74 \\
\hline $\begin{array}{c}+\gamma \\
\text { The proposed model: } \\
\mathrm{y}=-\frac{\alpha}{\delta}\left(1-\mathrm{e}^{-\beta \delta}\right)+\gamma\end{array}$ & $\begin{array}{c}2.378 \\
21\end{array}$ & $\begin{array}{c}0.308 \\
13\end{array}$ & $\begin{array}{c}1.453 \\
79\end{array}$ & 0.28 & $\begin{array}{c}17.51 \\
26\end{array}$ & $\begin{array}{c}0.057 \\
7\end{array}$ & $\begin{array}{c}1.188 \\
44\end{array}$ & 1.34 \\
\hline
\end{tabular}

Then, for many different sets of $P_{r}$ and $\omega, W$ versus $\delta$ is generated by DSMC $[12,13]$ and $\alpha, \beta$ and $\gamma$ are optimized. Note that we have $\alpha, \beta$ and $\gamma$ parameters that correspond to many different sets of $P_{r}$ and $\omega$. Finally, attempts are made to relate the $\alpha, \beta$ and $\gamma$ parameters to $P_{r}$ and $\omega$. As a result, the following equations 20-30 could be achieved.

$$
\begin{aligned}
& \alpha=a_{1} \omega^{2}+a_{2} \omega+a_{3} \\
& \beta=\frac{b_{1}}{\omega+b_{2}} \\
& \gamma=c_{1} \ln \left(\omega+c_{2}\right)+c_{3}
\end{aligned}
$$

Finally, the coefficients $a_{1}$ to $c_{3}$ are functions of the pressure ratio as follow:

$a_{1}=-0.04704 P_{r}^{3}+0.02769 P_{r}^{2}-0.02967$,

$a_{2}=-0.02640 P_{r}^{2}-0.14011 P_{r}+3.83918$

$a_{3}=-0.71279 P_{r}^{2}+2.96682 P_{r}+1.67151$

$$
\begin{aligned}
& b_{1}=-0.28262 P_{r}^{2}+0.06048 P_{r}+0.35171 \\
& b_{2}=-0.22249 P_{r}+1.27986 \\
& c_{1}=1.81730 P_{r}^{2}+0.13470 P_{r}-2.86718 \\
& c_{2}=29.28050 P_{r}^{2}-65.32088 P_{r}+90.59141 \\
& c_{3}=-7.46284 P_{r}^{2}-2.88368 P_{r}+14.44894
\end{aligned}
$$

\subsection{Predictions of the Proposed Model for Short Tubes}

Some model calculations were performed for various combinations of the variables. Figure 1 illustrates the reduced flow rate as a function of rarefaction parameter for different tube length to radius ratios as well as four pressure ratios. As can be seen, $W$ varies continuously sigmoidal with respect to $\delta$, and generally has an increasing trend with $\delta$. For the low and high ranges of rarefaction parameter, $\mathrm{W}$ increases very slowly, corresponding to the free molecular and hydrodynamic regions, respectively. In contrast, in the transition 
region the increases are steeper. As a limit for the reduced flow rate, when $\delta$ tends toward small values i.e. $(\delta \rightarrow 0)$, then $W$ approaches $1-P_{r}$ for an orifice ( $\omega \rightarrow 0)$, which is obviously observed from Figures 1a, 1b and 1c. For higher values of $P_{r}$ e.g. $P_{r}=0.9$, this limit is met by the model with a lower accuracy. From Figure 1a one can see that $W$ varies between 0.1 and 1.526. Barashkin
[7] measured $W$ for an orifice. and reported a $W$ value of 1.501 which is close to the value shown in Figure 1a. In general the lower $P_{r}$ or $\omega$, the higher the reduced flow rate. It is noted that according to the data points utilized for deriving the Equations (19) to (30), the proposed model is applicable for the following ranges: $0.01<\delta<1000$, $0.0<P_{r}<0.9$ and $0.01<\omega<20$.
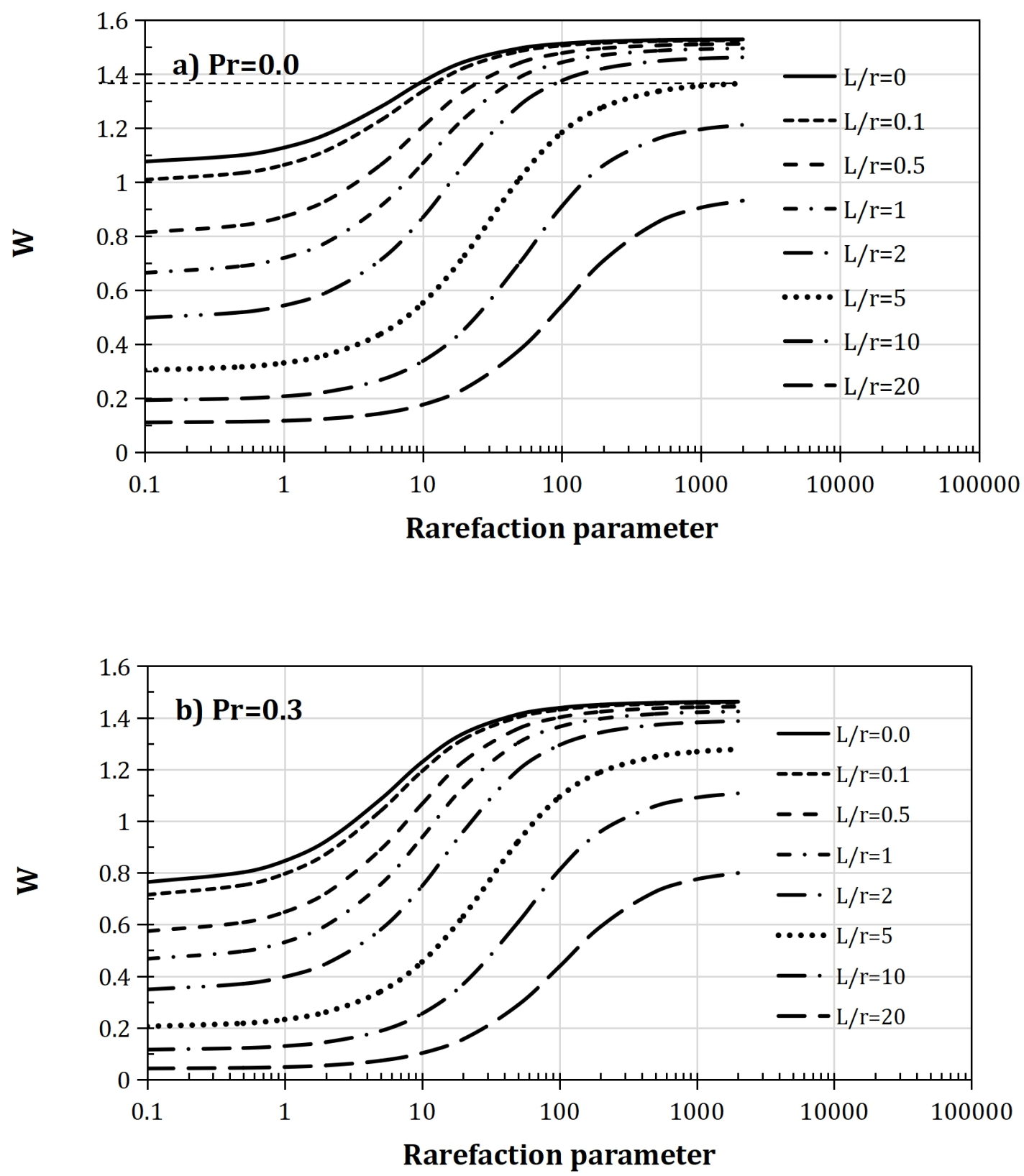

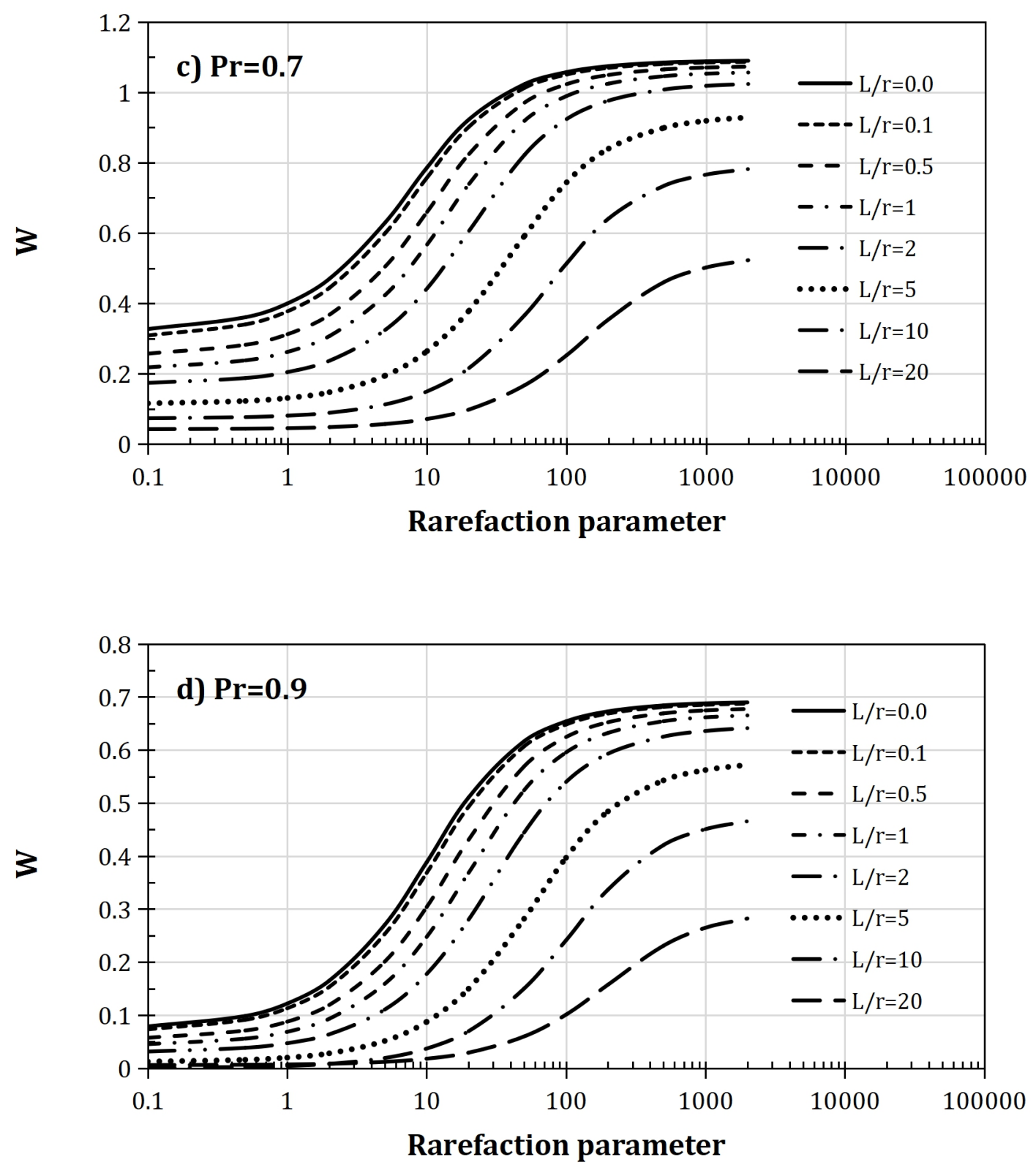

Figure 1 Reduced flow rate as a function of rarefaction parameter, tube length to radius ratio and pressure ratio a) $\operatorname{Pr}=0.0$, b) $\operatorname{Pr}=0.5$, c) $\operatorname{Pr}=0.7$ and d) $\operatorname{Pr}=0.8$

Theoretically, both $\quad P_{r} \quad$ illustrates that this limit was met by the and $\delta$ approach zero, $W$ tends toward proposed model. The agreement was $1 /(1+\omega / 2)$ [21]. Interestingly, Figure 2 more pronounced when $\omega>0.5$. 


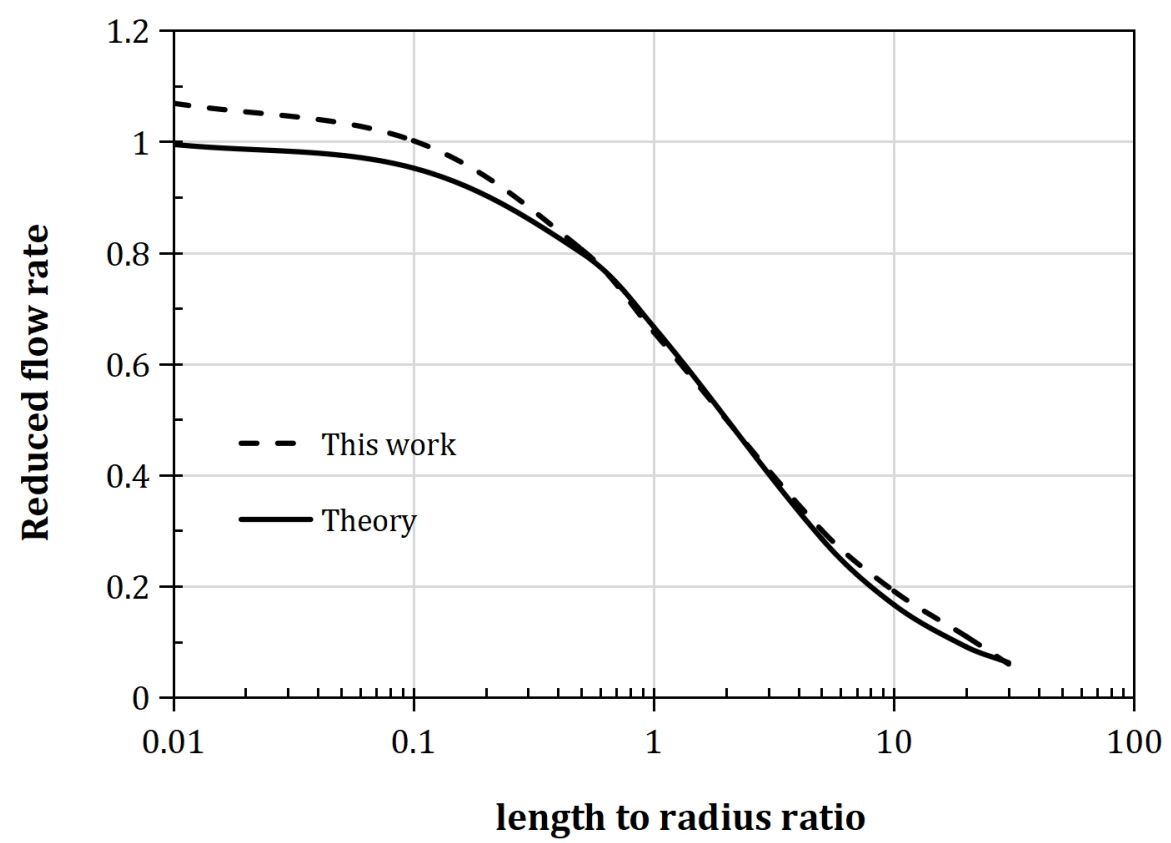

Figure 2 Reduced flow rate as a function of tube length to radius ratio (discharge to vacuum at low rarefaction parameter)

\subsection{Verification of the Proposed Model}

Figure 3 shows the comparison of the model predictions with the experimental data collected from several references $[4$, $5,6,7,8]$. There is a good agreement between the model and the experimental data (symbols) at low to moderate $\delta$ values up to 500 . The higher the $\omega$, the higher is the compatibility with the experimental points. Jitschin et al. reported $W=\sim 1.6$, which is higher than the predicted value of 1.50 , when $\omega=0$ and $\delta>100$, corresponding to the fully hydrodynamic regime [6] whereas, as already mentioned, Barashkin obtained an experimental value of 1.501 for this limit [7], while the predicted value is 1.526. At $\omega=2$, the model predicted the values higher than the experimental ones obtained by Barashkin [7] (in the range of $10<\delta<400$. Figure 3 clearly shows that the proposed model is capable to predict $W$ at $\omega$ as high as 25 in nearly the whole range of $\delta$.

Figure 4, depicts the model predictions versus the experimental data from Sreekanth [4] for $P_{r}$ values of 0.1 and 0.5 . The agreement of the predicted and the experimental values is satisfactory. Figure 5 exhibits this model prediction against the Fujimoto and Usami [5] model, which also represents their experimental data. The proposed model follows very well the trend of the data for $P_{r}$ values of 0.1 to 0.5 when $\omega=$ 1.0 and 2.6. There is some overestimation for the prehydrodynamic values of $\delta$ relative to the Fujimoto and Usami model. 


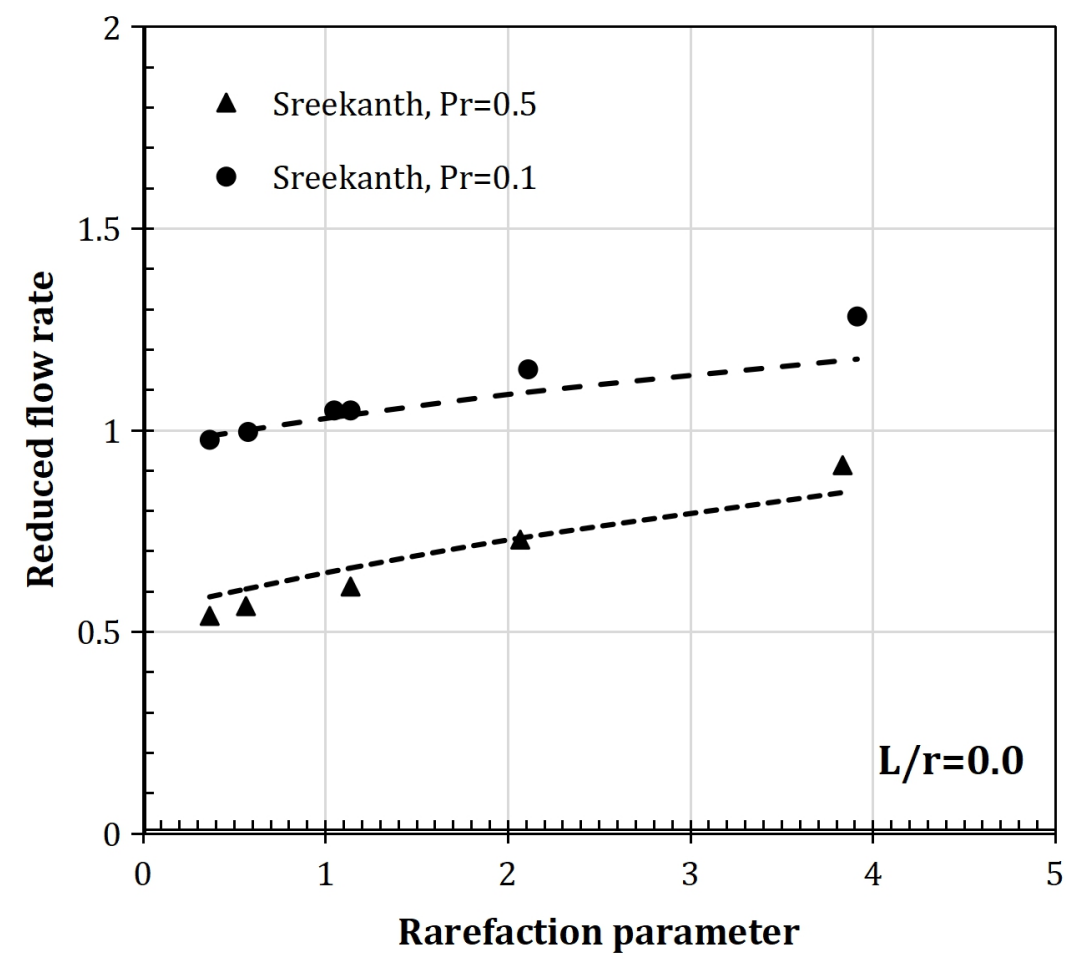

Figure 4 The model prediction of reduced flow rate versus the experimental data at $\omega=0$ [4]

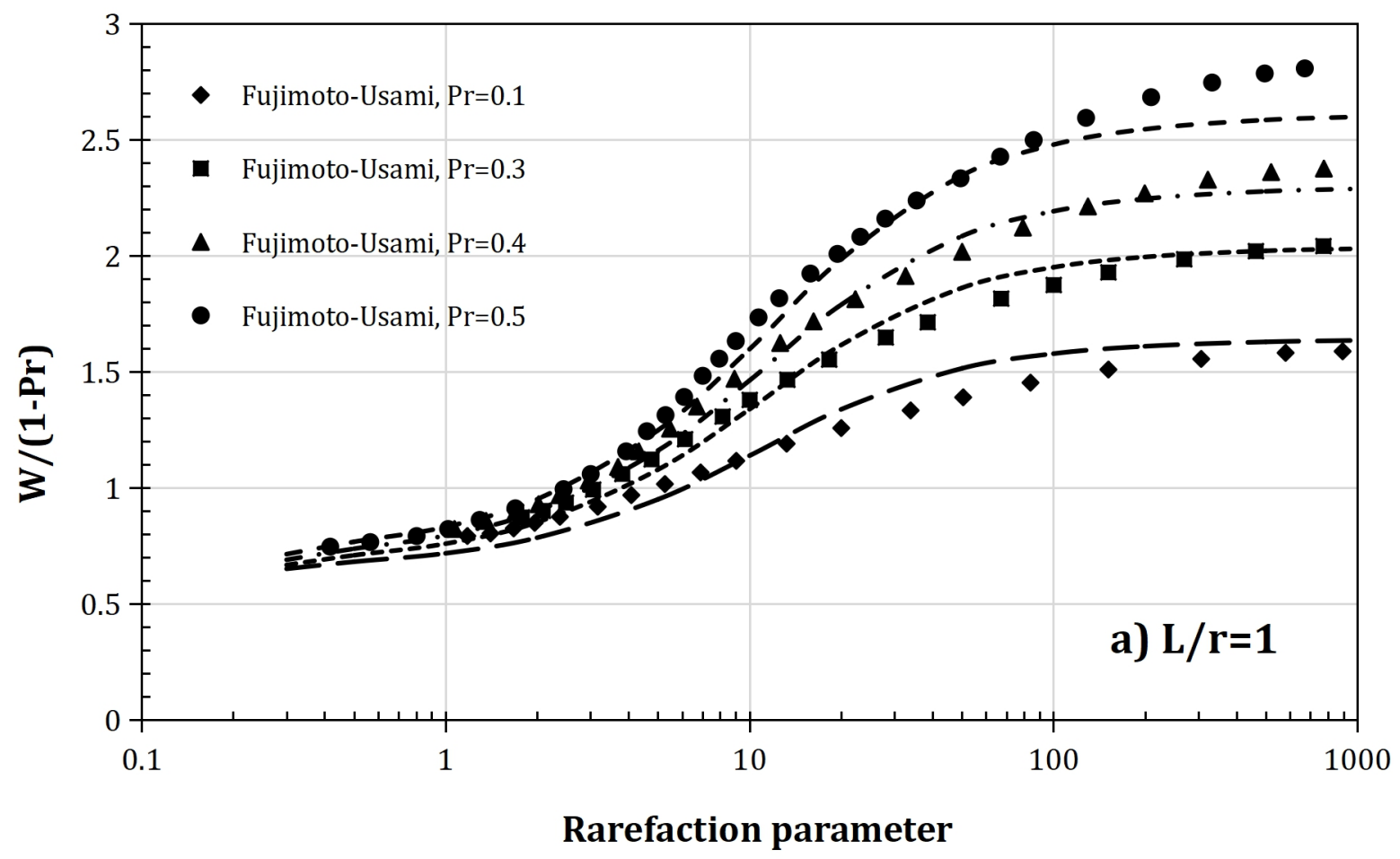




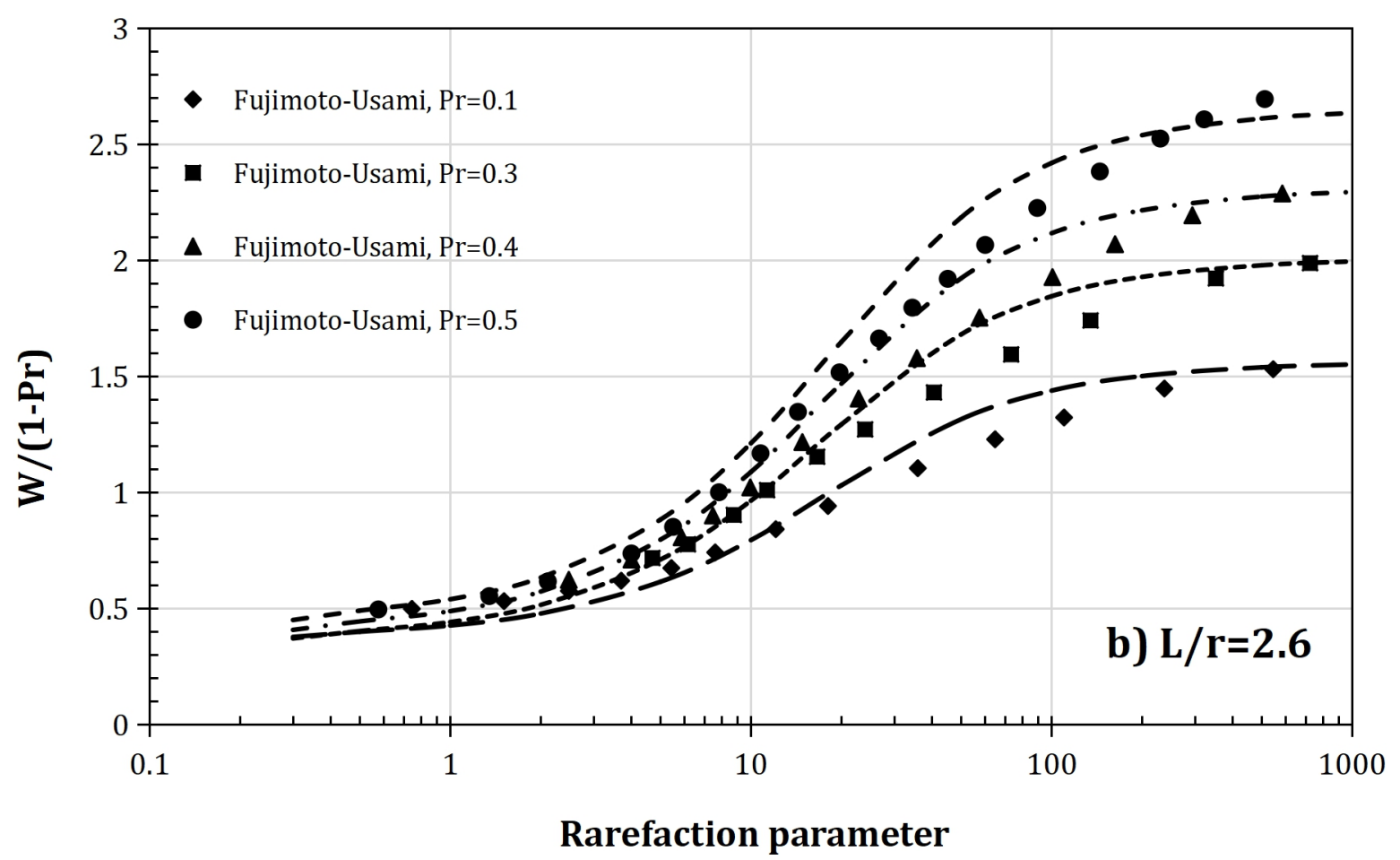

Figure 5 The model prediction of reduced flow rate versus the experimental data of Fujimoto and Usami, a) $\omega=1.0$, b) $\omega=2.6[5]$

Figure 6 compares the model prediction versus the values obtained by Varoutis et al. via DSMC [12, 13]. In the figure, the reduced flow rate is given as a function of pressure ratio for several different $\omega$ 's .From the figure, the trend is basically linear when the rarefaction parameter is as low as 1 for all $\omega\left(L_{p} / r_{p}\right)$ values given in Figures $6 a-c$ at $\omega=0.1$ (Figure 6a), the model prediction underestimates $W$ for $10<\delta<100$. However, there is an acceptable agreement between the model and the DSMC predictions for the whole range of $0.0<P_{r}<0.9$. Figure 7 shows the model predictions compared with the DSMC data, for $\omega=0.1,5$ and 10. Generally, there is a good agreement between the two approaches. However, at high values of the rarefaction parameter $(\delta>100)$, the agreement is fairly poor for $\omega=5$ and 10 . From Figure $7 \mathrm{a}$, it can be seen that at low values of $\omega$ (i.e. $\omega=0.1$ ), as $\delta$ approaches zero (free molecular regime), $W$ tends toward 1- $P_{r}$. The model almost meets this limit up to $P_{r}<0.9$, hence, there is a good agreement between the model and the DSMC data in this regard. 


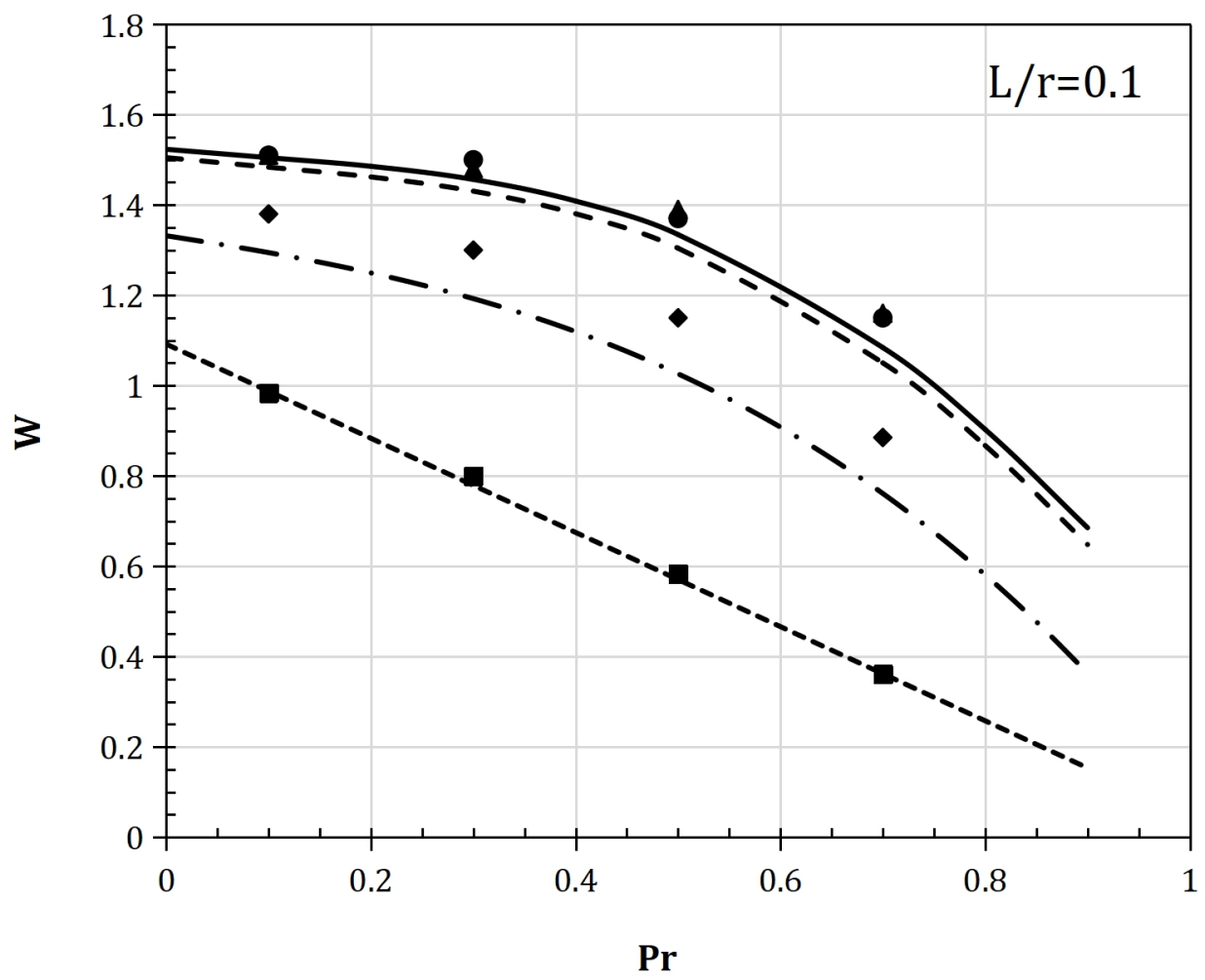

- DSMC-Rare. parameter $=1$

- DSMC-Rare. parameter $=10$

- DSMC-Rare. parameter $=100$

\ DSMC-Rare. parameter $=1000$

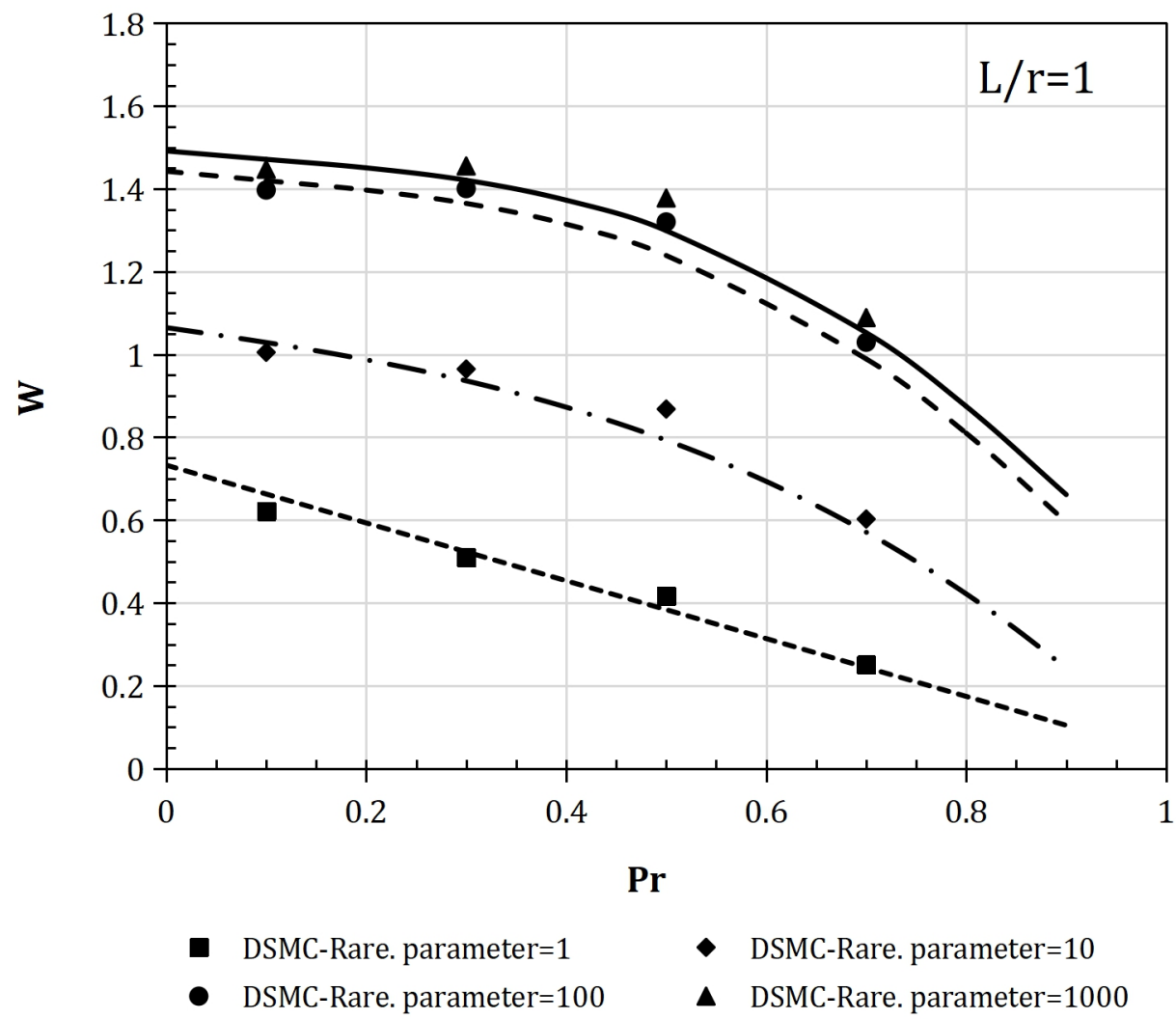




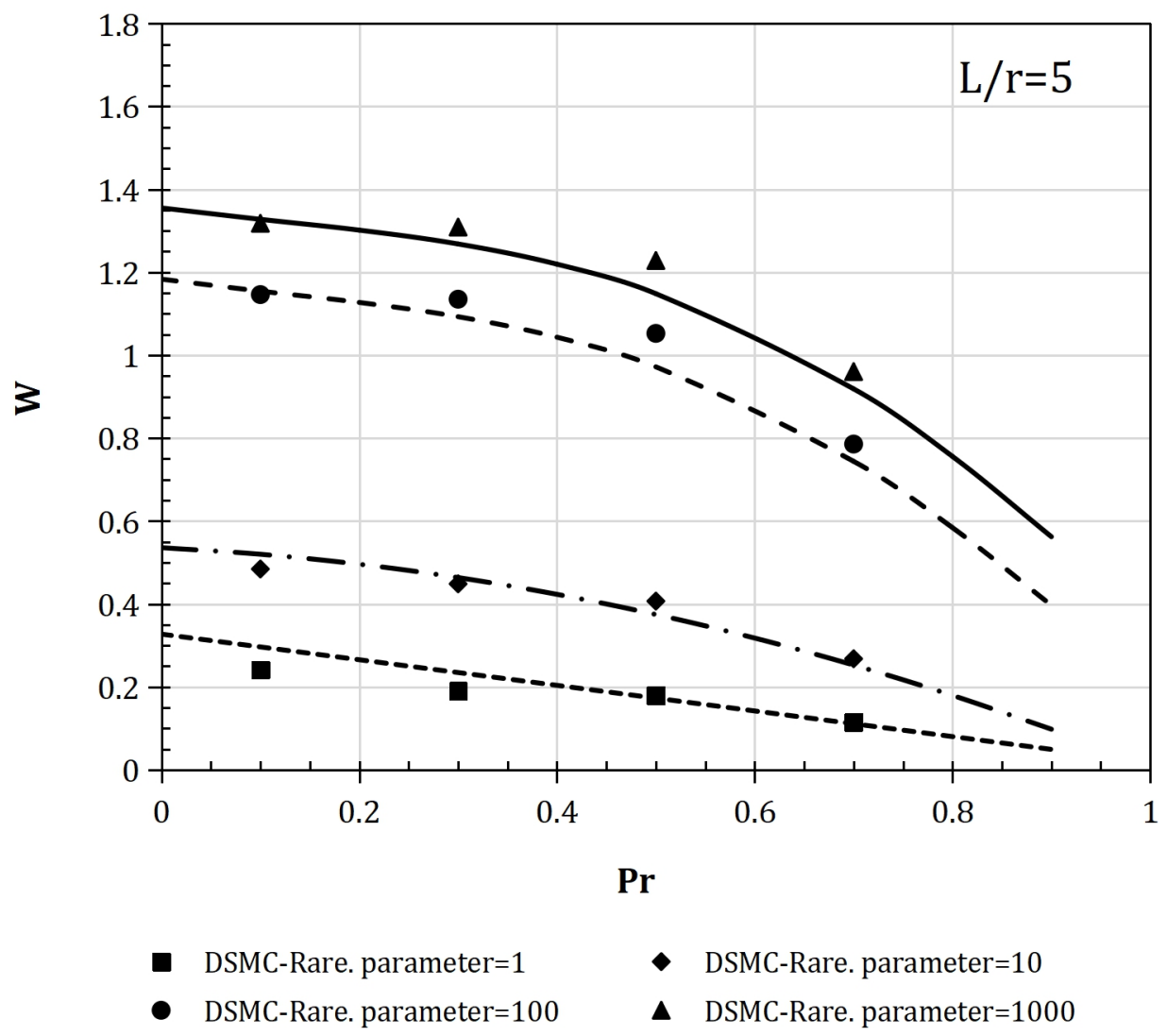

Figure 6 The model prediction of reduced flow rate versus the DSMC data of Varoutis et al. [12]: Reduced flow rate as a function of pressure ratio

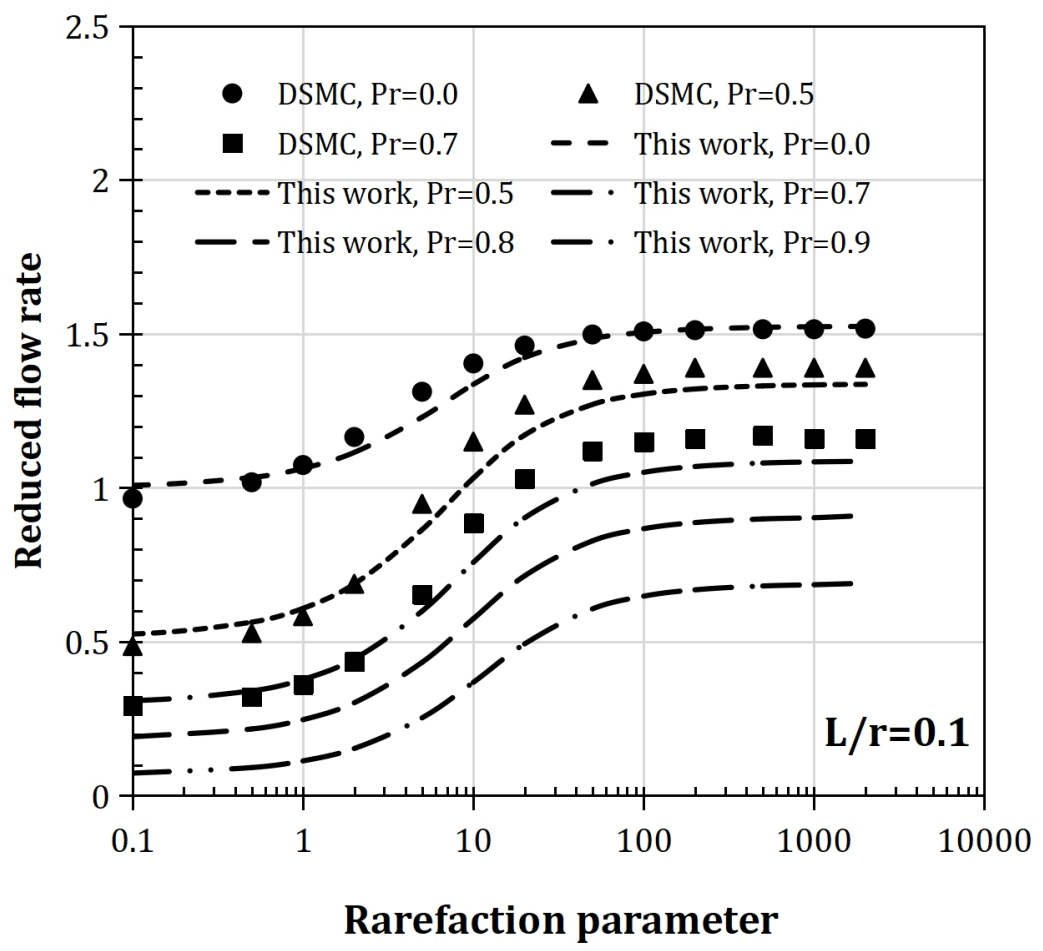



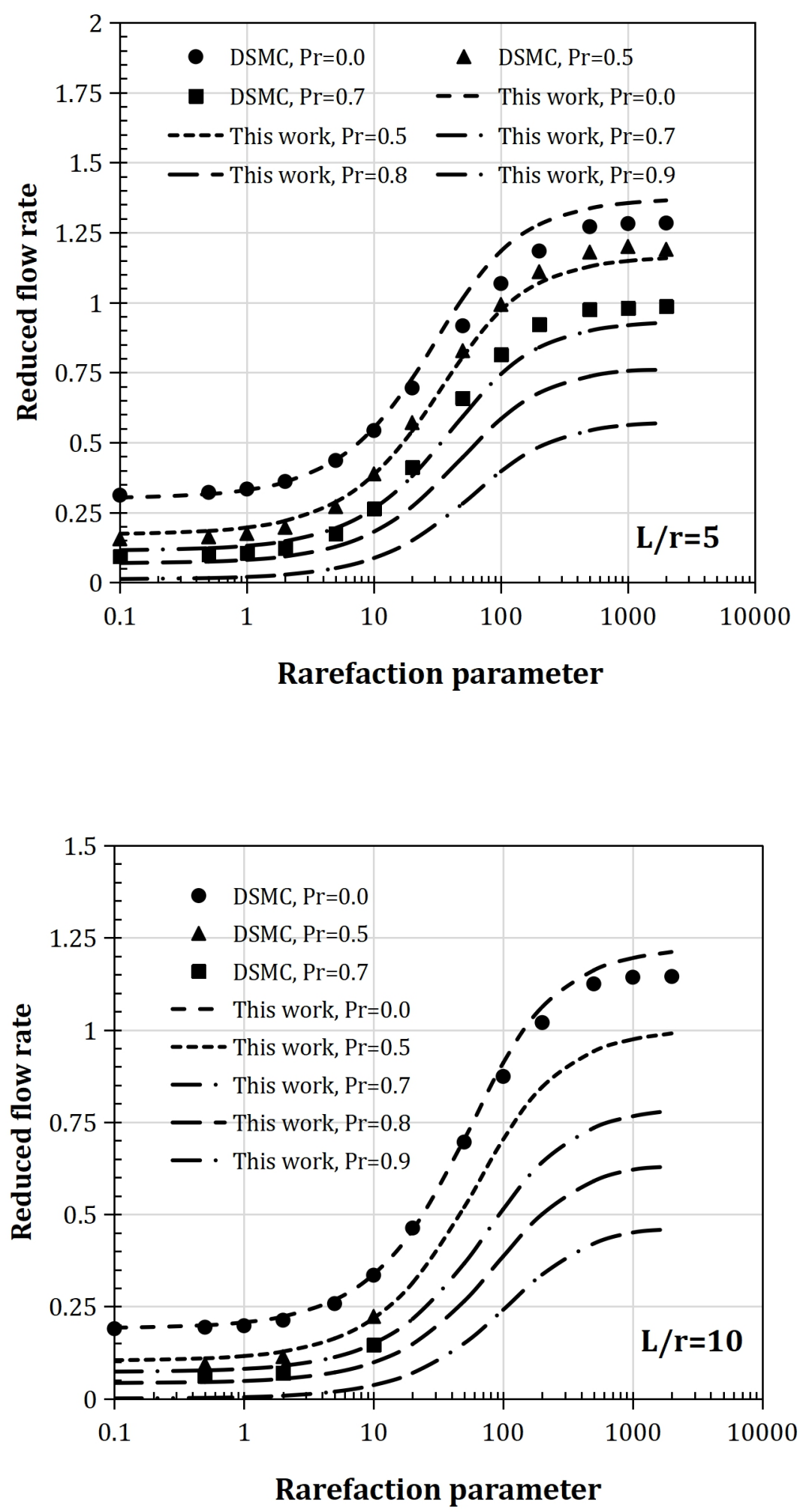

Figure 7 The model prediction of reduced flow rate versus the DSMC data of Varoutis et al. $[12,13]$ : Reduced flow rate as a function of rarefaction parameter 


\subsection{CONCLUSIONS}

A new model to predict the gas flow rate through short tubes under rarefied coditon was developed based on the sigmoidal bahaviour of reduced gas flow rate versus rarefaction parameter. The newly proposed model has the following advantages. The model can predict the gas flow rate as the functions of rarefaction parameter, $\delta$, length to radius ratio, $\omega$, and pressure ratio, $\mathrm{Pr}$, at the upstream and downstream of the micro/nano short tubes. Therefore the proposed model is able to exhibit all the trends of the reduced flow rate that occur when the major variables are changed. Intrestingly the model meet almost all the criteria of a rarefied gas flow system, moreover, the proposed model can predict the reduced flow rate of rarefied systems, not only at the free molecular and the hydrodynamic region, but also at the transition region, hence covering all the Knudsen number domain within the utilised data. There is a high degree of agreement between the model predictions and the published experimental data. In conclusion, the proposed model provides a useful tool to make a valid prediction of the rarefied gas flow behavior in a wide range of gas transport regime.

\section{ROMAN LETTERS}

$a$

model parameter

$A_{m} \quad$ effective membrane area

$A_{t p} \quad$ total cross sectional area of

the pores

b model parameter

c model parameter

$K_{n} \quad$ the Knudsen number

$l_{p} \quad$ tube/pore depth

$M \quad$ gas molecular weight

$F \quad$ mass flow rate of the system

$F_{f m 0} \quad$ mass flow rate through an orifice into vacuumfor one pore

$\begin{array}{ll}F_{f i m} & \text { model parameter } \\ P_{0} & \text { upstream pressures } \\ P_{I} & \text { downstream pressures } \\ P_{r} & \text { pressure ratio } \\ R & \text { the universal gas constant } \\ R_{e} & (8.314 \mathrm{~J} \mathrm{~mol}-1 \mathrm{~K}-1) \\ r_{p} & \text { the Reynolds number } \\ t & \text { tube radius } \\ T_{0} & \text { permeation time } \\ V & \text { upstream absolute temperature } \\ & \text { volume of gas permeated through the } \\ v_{m} & \text { membrane }\left(25^{\circ} \mathrm{C}, 1 \text { bar }\right) \\ W & \text { most prabable gas velosity } \\ & \text { reduced flow rate }\end{array}$

\section{GREEC LETTERS}

$\begin{array}{ll}\alpha & \text { parameters as a functions of pressure ratio } \\ \beta & \text { parameters as a functions of pressure ratio } \\ \delta & \text { rarefaction parameter } \\ \varepsilon & \text { surface porosity } \\ \phi & \text { model parameter } \\ \gamma & \text { parameters as a functions of pressure ratio } \\ \lambda & \text { gas mean free path } \\ \mu_{0} & \text { gas viscosity at upstream conditions } \\ \omega & \text { tube depth to radius ratio }\end{array}$

\section{ACKNOWLEDGEMENT}

The authors gratefully acknowledge Persian Gulf University for their financial support.

\section{REFERENCES}

[1] C. M. Ho, Y. C. Tai. 1998. Micro-electro-mechanicalsystem (MEMS )and Fluid Flows. Annu Rev Fluid Mech. 30: 579- 
612.

[2] A. Beskok, G. E. Karniadakis. 1994. Report: A Model for Flows in Channels, Pipes, and Ducts at Micro and Nano Scales. Microscale. Therm. Eng. 3: 4377.

[3] H. W. Liepmann. 1961. Gas Kinetics and Gas Dynamics of Orifice Flow. J Fluid Mech. 10: 65-79.

[4] A. K. Sreekanth. 1965. Transition Flow Through Short Circular Tubes. Phys. Fluids. 8: 1951-1956.

[5] T. Fujimoto, M. Usami. 1984. Rarefied Gas Flow Through a Circular Orifice and Short Tubes, J. Fluids Eng. 106: 367-373.

[6] W. Jitschin, M. Ronzheimer, and S. Khodabakhshi. 1999. Gas Flow Measurement by Means of Orifices and Venturi Tubes. Vacuum. 53: 181-185.

[7] S. T. Barashkin. 1997. Experimental Investigation of Flows of Gas into Vacuum. Ph.D. Thesis. Ural State Technical University.

[8] Marino. 2009. Experiments on Rarefied Gas Flows Through Tubes. Microfluid Nanofluid. 6: 109-119.

[9] A. A. Alexeenko, S. F. Gimelshein, D. A. Levin, A. D. Ketsdever, M. S. Ivanov. 2002. Study of Orifice Flow in the Transitional Regime. 21st International Symposium on Rarefied Gas Dynamics AIP Conference Proceedings. 663: 565-571.

[10] A. A. Alexeenko, S. F. Gimelshein, D. A. Levin, A. D. Ketsdever, M. S. Ivanov. 2003. Measurements and Simulation of Orifice Flow for Micropropulsion Testing. $J$ Power Propuls. 19: 588-594

[11] T. C. Lilly, S. F. Gimelshein, A.
D. Ketsdever, G. N. Markelov. 2006. Measurements and Computations of Mass Flow and Momentum Flux Through Short Tubes in Rarefied Gases. Phys Fluids. 18: 1-11.

[12] S. Varoutis, D. Valougeorgis, O. Sazhin, F. Sharipov. 2008. Rarefied Gas Flow Through Short Tubes into Vacuum. J Vac. Sci. Technol A. 26: 229-238.

[13] S. Varoutis, D. Valougeorgis, F. Sharipov. 2009. Simulation of Gas Flow Through Tubes of Finite Length Over the Whole Range of Rarefaction for Various Pressure Drop Ratios. J. Vac. Sci. Technol. A 27: 1377-1391.

[14] W. Wagner. 1992. A Convergence Proof for Bird's Direct Simulation Monte Carlo Method for the Boltzmann Equation. J. Stat. Phys. 66: 1011-1044.

[15] M. D. Liu, K. J. Xu, S. Horpibulsuk. 2015. A Mathematical Function to Represent S-shaped Relationships for Geotechnical Applications. ICE Proceedings. 166: 321-327.

[16] D. Kucharavy, R. De Guio. 2011. Application of S-shaped Curves. Procedia Eng. 9: 559-572.

[17] M. Knudsen. 1909. Die gesetze der molekularströmung und der inneren reibungsströmung der gase durch röhren. Annalen der Physik. 28: 75-130.

[18] R. Byron Bird, Warren E. Stewart, Edwin N. Lightfoot. 2007. Transport Phenomena. Revised 2nd Edition. John Wiley $\&$ Sons.

[19] F. Sharipov. 2008. Encyclopedia of Microfluidics and Nanofluidics. Springer-Verlag, New York.

[20] S. Roy, R. Raju, H. F. Chuang, B. A. Cruden, and M. Meyyappan. 
2003. Modeling Gas Flow Through Micro Channels and Nano Pores. J. Appl. Phys. 93: 4870-4879.

[21] W. Steckelmacher. 1986. Knudsen Flow 75 Years on: The Current State Of The Art For Flow Of Rarefied Gases In
Tubes and Systems. Rep. Prog. Phys. 49: 1083-1107.

[22] F. Sharipov. 2001. Rarefied Gas Flow Through a Thin Orifice, Rarefied Gas Dynamics. 22nd International Symposium, AIP Conf. Proc. 585: 494-501. 